treatment is recommended to preserve motor function in ambulant children with DMD. (Beenakker EAC, Fock JM, Van Tol MJ et al. Intermittent prednisone therapy in Duchenne muscular dystrophy. Arch Neurol Jan 2005;62:128-132). (Respond: Ernesto AC Beenakker MD, Department of Neurology, University Hospital Groningen, Hanzeplein 1, 9700 RB Groningen, the Netherlands).

COMMENT. In previous studies of the effects of prednisone in DMD, a significant increase in muscle strength, pulmonary function, and functional ability has been demonstrated in a randomized controlled trial in 99 boys treated at the University of Rochester, NY (Griggs RC et al. Arch Neurol 1991;48:383-388; Ped Neur Briefs April 1991). Improvement was rapid, occurring in 10 days and was maximal after 2 months. A dose of $0.75 \mathrm{mg} / \mathrm{kg} /$ day was recommended in patients who experience functional decline. In another report (Fenichel GM et al. Neurology 1991;41(Suppl 1):166), the beneficial effects of prednisone, $0.65 \mathrm{mg} / \mathrm{kg} /$ day, extending over a two year observation period were demonstrated in 89 boys with DMD. See Ped Neur Bricfs June 1991 for further articles on prednisone in DMD and its effect on immunological mechanisms.

\title{
CEREBRAL MALFORMATIONS
}

\section{SPECTRUM OF CORPUS CALLOSUM AGENESIS}

Magnetic resonance imaging and clinical features of 16 children with agenesis of the corpus callosum (ACC) are reviewed at UAE University, United Arab Emirates. Three groups of patients were recognized: Group 1). 8 patients without involvement of other brain areas, 3 having lipomas, or interhemispheric cyst, 3 with partial ACC, 5 with Probst bundles (aberrant longitudinal fiber bundles on medial hemispheral walls), and 4 with epilepsy; Group 2). 4 patients with ACC complicated by severe telencephalic dysgenesis (periventricular heterotopia, microcephaly with cortical dysplasia, microlissencephaly and cerebellar hypoplasia, diffuse agyria-pachygyria and pontocerebellar dysplasia), absent Probst bundles, and developmental delay and epilepsy in 3; Group 3). 4 children with ACC as part of a syndrome: 1 with Aicardi syndrome, 2 with L1 disease and mutations in the L1CAM gene, and 1 with Mowat-Wilson syndrome with mutation in the zinc finger homeo box 1B (ZFHX1B) gene; all 4 had severe developmental delay and mental retardation and 1 had infantile spasms. Severe handicaps, developmental deiay, mental retardation, and neurologic deficit occurred in patients of groups 2 and 3. Two of the 8 patients in group 1 were developmentally delayed, mentally retarded, and had neurologic deficits. Parental consanguinity was present in 7 of the 16 patients, and genetic factors are involved in ACC cases associated with various syndromes. (Sztriha L. Spectrum of corpus callosum agenesis. Pediatr Neurol February 2005;32:94-101). (Respond: Dr Sztriha, Department of Pediatrics, Faculty of Medicine and Health Sciences, UAE University, POB 17666, Al Ain, United Arab Emirates).

COMMENT. MRI is important in the evaluation of developmental delay or epilepsy. ACC is also recognized on prenatal ultrasound after the $20^{\text {th }}$ week. Dobyns describes true types and secondary types of ACC (Am J Hum Genet 1996;58:7-16). Those associated with major malformations or degeneration are considered secondary. 